\title{
Unreality of the Cross-Over between Regions II and III, and on a New Region in the T-C Diagram of Polymer Solutions
}

\author{
Tomo-o OYAma, Kohzoh SHIOKAWA, \\ and Koumei BABA \\ Department of Applied Science, Faculty of Engineering, \\ Kyushu University, Hakozaki, Higashi-ku, \\ Fukuoka 812, Japan
}

(Received December 26, 1981)

\begin{abstract}
KEY WORDS T-C Diagram / Cross-Over / Semi-Dilute / Tricritical / Correlation Length / Light Scattering / Polystyrene /
\end{abstract}

Daoud and Jannink ${ }^{1}$ have used the scaling theory to show that polymer solutions may be divided into five regions (Regions I, I', II, III, and IV) each with its own particular character. Their theory gives cross-over relations between the regions and the T$\mathrm{C}$ diagram of polymer solutions. $\mathrm{We}^{2}$ also derived similar results using the mean field theory which includes the third virial coefficient and is based on intuitive assumption.

Some of these theoretical cross-over relations have been confirmed experimentally, but the crossover relation between Regions II (semi-dilute region) and III (concentrated tricritical region), given by $\tau \sim C^{* *}$ ( $\tau=$ reduced temperature, $C^{* *}=$ crossover concentration), will require additional research for clarification.

$\mathrm{We}^{3}$ constructed a new light scattering photometer which allows the radius of gyration of a polymer chain at infinite dilution ( $\equiv$ the correlation length at $C=0$ ) to be measured down to $\sim 5 \mathrm{~nm}$ from the angular dependence of scattering intensity. This angular dependence at a finite concentration gives the correlation length $\xi$ at that concentration. (The right-hand side of eq 5 in the previous paper ${ }^{3}$ is generalized to $\left(8 \pi^{2} / 3 \lambda^{2}\right) \xi^{2} \cos ^{2} \theta$, when $\xi \ll \lambda$.)

In this paper, new results on the cross-over relation between Regions II and III observed through $\xi$ are reported.

\section{EXPERIMENTAL RESULTS}

The light scattering photometer, the standard polystyrene samples and the experimental procedure used in this study are the same as those in the previous paper. The results of this study are reported separately, in datail.

Figure 1 shows the concentration dependence of $\xi^{2}$ in benzene solutions of different molecular weight samples of polystyrene at $30^{\circ} \mathrm{C}$. It is seen that $\xi^{2}$ is independent of molecular weight in the region of higher concentration and that the theoretical relation for Region II, $\xi^{2} \sim C^{-3 / 2}$, fits the data points.

Figure 2 shows $\xi^{2}$ in cyclohexane solutions of the F-40 sample $\left(M_{w}=4 \times 10^{5}\right)$ at and above the $\Theta$ temperature $\left(34.5^{\circ} \mathrm{C}\right) . \xi^{2}$ decreases gradually with increasing polymer concentration. However, the theoretical relations for Region II, $\xi^{2} \sim C^{-3 / 2}$, and Region III, $\xi^{2} \sim C^{-2}$, can not be seen from this figure even at the highest concentration measured. Measurements at higher concentrations were not feasible, because of the great difficulty of preparing such solutions.

In Figure 3, our results (the solid lines A, B, and C) are shown together with the results of Cotton et $a l .{ }^{4}(\mathrm{O})$ obtained by neutron scattering in a carbon disulfide solution. The agreement between the two results is quite well. The slopes of the dashed lines for the extrapolation of actual data were chosen as 


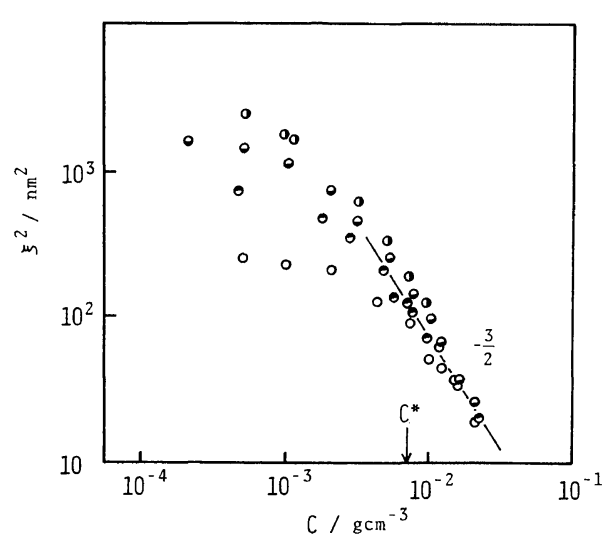

Figure 1. Double logarithmic plots of the correlation length $\xi^{2}$ vs. concentration $C$ for the benzene solutions. Sample molecular weights: $\bigcirc, 1.86 \times 10^{5}$ (F20); $\Theta$, $4.22 \times 10^{5}(\mathrm{~F} 40) ; \theta, 7.75 \times 10^{5}(\mathrm{~F} 80) ; \quad$, $1.26 \times 10^{6}$ (F126). The straight line represents the theoretical slope of $-3 / 2$ for Region II. $C^{*}$ is the calculated value of the cross-over concentration for F40 between Regions I and II.

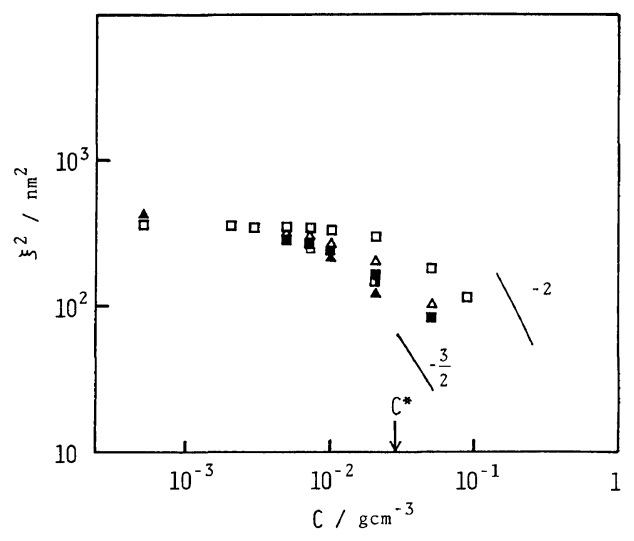

Figure 2. Double logarithmic plots of the correlation length $\xi^{2} v s$. concentration $C$ for the F40-cyclohexane solutions at and above the $\Theta$ temperature: $\square, 34.5^{\circ} \mathrm{C}$; $\triangle, 39.7^{\circ} \mathrm{C} ; \boldsymbol{\square}, 44.7^{\circ} \mathrm{C} ; \mathbf{\square}, 49.5^{\circ} \mathrm{C} ; \boldsymbol{\Delta}, 54.5^{\circ} \mathrm{C}$. The straight lines represent the theoretical slope of -2 for Region III and $-3 / 2$ for Region II. $C^{*}$ is the calculated value of the cross-over concentration at the $\Theta$ temperature between Regions I' and III.

-2 for the cyclohexane solution at $34.5^{\circ} \mathrm{C}$ (Region III), and as $-3 / 2$ for the other solutions (Region II). Although the location of the lines for cyclohexane solutions and the slope of the line for the cyclohexane solution at $39.7^{\circ} \mathrm{C}$ are somewhat arbitrary, this arbitrariness had no effect on the follow-

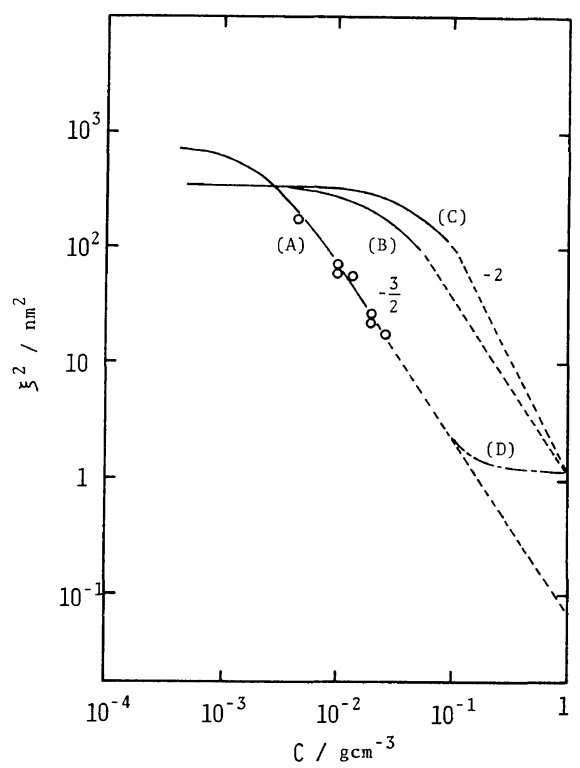

Figure 3. Double logarithmic plots of correlation length $\xi^{2}$ vs. concentration $C$ : the solid line $\mathrm{A}, \mathrm{F} 40$ benzene; B, F40-cyclohexane at $39.7^{\circ} \mathrm{C} ; \mathrm{C}, \mathrm{F} 40$ cyclohexane at $34.5^{\circ} \mathrm{C} ; \mathrm{O}$, neutron scattering. ${ }^{4}$ The broken lines represent extrapolated lines. The dot-dash line $\mathrm{D}$ represents a new region (see text).

ing semi-quantitative considerations.

A simple derivation of the relation between $\xi$ and $\alpha$ in Regions II and III is given below. According to the scaling theory, ${ }^{5}$ a pair correlation function $g(r)$ is $\sim N / r^{3}$. Using the relation $r^{2} \sim \alpha^{2} N, g(r)$ is $\sim 1 /$ $r \alpha^{2}$, where $\alpha$ is the Flory's expansion factor. Since $g(\xi) \sim C$ in Regions II and III,

$$
\xi \sim \frac{1}{C \alpha^{2}}
$$

is easily derived. Since $\alpha=1$ in Region III, and $\alpha>1$ in Region II, $\xi$ in the former region is always larger than that in the latter region. The results in Figures 1 and 3 agree with this prediction.

As for Figure 3, two problems may be pointed out. First, the respective lines (C) and (B) for Region III and for the cyclohexane solution at $39.7^{\circ} \mathrm{C}$ merge at a $C$ near $C=1$. On the other hand, the respective lines (C) and (A) for Region III and for the benzene solution do not intersect with each other at $C=1$. This implies that the existence of the cross-over between Regions II and III does not occur in the real concentration range $\left(C^{* *}<1\right)$. Second, the values of $\xi$ extrapolated to $C=1$ are 
ca. $1 \mathrm{~nm}$ and $c a .0 .3 \mathrm{~nm}$ for the cyclohexane solutions and for the benzene solution, respectively. The latter value is too low compared to the size of a polystyrene segment in the melt, since the diameter of a monomer calculated from the amorphous density is $c a .0 .7 \mathrm{~nm}$.

\section{THEORETICAL CONSIDERATIONS}

From our theory, ${ }^{2}$ the following equation was derived for Regions II and III,

$$
\alpha^{8}-\alpha^{6} \sim\left(\frac{\beta_{2}}{C}\right)+\left(\frac{\beta_{3}}{C}\right) N^{-1 / 2} \alpha^{-3}
$$

where $\alpha$ is the Flory's expansion factor; $\beta_{2}$ is proportional to the second virial coefficient of a segment and the reduced temperature $\tau, \beta_{3}$ is proportional to the third virial coefficient and was assumed to be a positive constant and of the order of unity in the regions considered, $N$ is the number of segments in a polymer chain. Equation 2 is equivalent to eq 9 in our previous paper, in which the second term on the right side of eq 2 was omitted because only the limit of $N \rightarrow \infty$ was considered.

At the limit of $N \rightarrow \infty$, eq 2 suggests a crossover behavior between the $\beta_{2} / C \gg 0$ region or Region II (at this time, $\alpha^{2} \gg 1$ and the $\alpha^{6}$ term is neglected) and the $\beta_{2} / C=0$ region or Region III. The cross-over condition is that $\alpha$ in Region II $\left(\alpha^{8} \sim \beta_{2} / C\right)$ is equal to $\alpha$ in Region III $(\alpha=1)$, and the relation $\beta_{2} \sim \tau \sim C^{* *}$ is derived. However, a solution with $\beta_{2}>0$ has the character of Region III only at the unreasonable limit of $C \rightarrow \infty$. If $C^{* *}$ appears in the actual concentration range $\left(C^{* *}<1\right)$, the value of $\beta_{2}$ must be of the order of $10^{-1}$ or less and the solution has the character of Region II in a range of $C \sim 10^{-2}$ of less, since $\beta_{2} / C \gg 0$ in Region II.

For finite $N$, the effect of the $\beta_{3}$ term in eq 2 must be taken into consideration. In order to maintain the character or Region II, the additional condition $\beta_{2} \gg \beta_{3} N^{-1 / 2} / \alpha^{3}$ is necessary. This condition is simplified to $\beta_{2} \gg N^{-1 / 2}$ near $\tau=0$, when $\beta_{3} / \alpha^{3} \sim 1$. When $\beta_{2} \sim 10^{-1}$ or less, $N$ must be $10^{4}$ or more. This value of $N$ corresponds to a molecular weight of $10^{7}$ or more for polystyrene provided the number of monomers in a segment is 10 . However, the solution of $N \sim 10^{4}$ does not clearly show the character of Region II, since $C \sim 10^{-2}$ is of the same order as the cross-over concentration between Regions I and II near $\tau=0$.

The values of $\tau$ for good solvent systems are considered to be of the order of unity or more. The experimental value of $\tau$ for the benzene solution in this study is 1.9 , as estimated from the $\Theta$ temperature, $106 \mathrm{~K}$, obtained from our study for very dilute solutions (the details will be reported in a separate paper). Accordingly, although good solvent systems show the character of Region II, their values of $C^{* *}$ are not reasonable.

In short, $C^{* *}$ is unreasonable, except for solutions of extremely high molecular weight samples in a narrow range near $\tau=0$.

Next, an explanation is made as to why the value of $\xi$ extrapolated to $C=1$ is too low for good solvent systems. According to eq 2 , the value of $\xi$ at $C=1$ for good solvent systems is lower than that extrapolated, since the effect of the $\alpha^{6}$ term is not negligible at higher concentrations. The higher the value of $\beta_{2}$, the lower the values of $\xi$ extrapolated to and at $C=1$ become. On the other hand, since $\alpha=1$ for the melt, $\xi$ at $C=1$ must converge to a definite value regardless of the value of $\beta_{2}$. This value may be obtained for the $\tau=0$ and $N \rightarrow \infty$ solution. (The extrapolated value for the curve $(C)$ in Figure 3 includes the effects of molecular weight and arbitrariness. The true value will be somewhat higher.)

These facts suggest that concentrated good solvent systems change from Region II not to Region III but to a new unknown region, where $\xi$ is either constant or less dependent on concentration than in Region II, as shown by the dot-dash line (D) in Figure 3, and the correlation function $g(r)$ near the origin will increase abruptly. Further research on $g(r)$ and the segment size should carried out in this study.

The mean field theory derived by Edwards and Jeffers $^{6}$ has provided relations different from those derived by the scaling theory or by us. According to their theory, $\xi^{2} \sim C^{-6 / 5} \tau^{-4 / 5}$ in semi-dilute solutions and $\xi^{2} \sim C^{-1} \tau^{-1}$ in the limit of $C \rightarrow \infty$. However, these relations were not confirmed by our experiments.

\section{REFERENCES}

1. M. Daoud and G. Jannink, J. Phys. (Paris), 37, 973 (1976).

2. T. Oyama and K. Shiokawa, Polym. J., 12, 507 (1980).

3. T. Oyama, K. Shiokawa, and K. Baba, Polym. J., 13, 
T. Oyama, K. Shiokawa, and K. Baba

821 (1981).

4. J. P. Cotton, B. Farnoux, and G. Jannink, J. Chem. Phys., 57, 290 (1972).

5. For example, P. G. de Gennes, "Scaling Concepts in
Polymer Physics," Cornell University Press Ltd., London, 1979, p 37.

6. S. F. Edwards and E. F. Jeffers, J. Chem. Soc. Faraday Trans. 2, 75, 1020 (1979). 